\title{
Fully Equipped Dynamic Model of a Bus
}

\author{
I. Kowarska, ${ }^{1}$ J. Korta, ${ }^{1}$ K. Kuczek, ${ }^{2}$ and T. Uhl ${ }^{1}$ \\ ${ }^{1}$ Department of Robotics and Mechatronics, AGH University of Science and Technology, Aleja Mickiewicza 30, \\ 30-059 Krakow, Poland \\ ${ }^{2}$ EC Engineering Sp. z o.o., Ulica Opolska 100, 31-323 Krakow, Poland \\ Correspondence should be addressed to T. Uhl; tuhl@agh.edu.pl
}

Received 26 June 2013; Accepted 5 February 2014; Published 15 July 2014

Academic Editor: Nuno Maia

Copyright (C) 2014 I. Kowarska et al. This is an open access article distributed under the Creative Commons Attribution License, which permits unrestricted use, distribution, and reproduction in any medium, provided the original work is properly cited.

Nowadays, the time to market a new vehicle is crucial for every company as it is easier to meet the customers' needs and expectations. However, designing a new vehicle is a long process which needs to take into account different performances. The most difficult is to predict a dynamic behavior of a vehicle especially when such a big vehicles as urban buses are considered. Therefore, there is a necessity to use a virtual model to investigate different performances. However, there is a lack of urban bus models that can fully reflect a dynamic behavior of the bus. This paper presents a fully equipped urban bus model which can be used to study a dynamic behavior of such vehicles. The model is based on innovative technique called cosimulation, which connects different modeling techniques (3D and $1 \mathrm{D})$. Such a technique allows performing different analyses that require small deformations and large translations and rotations in shorter time and automatic way. The work has been carried out in a project EUREKA CHASING.

\section{Introduction}

In nowadays vehicle industry, time to market is a crucial parameter. It is caused by an increasing number of customers' needs and expectations to be met. However, vehicle design is a long process, during which engineers need to take into account many guidelines and fulfill all the requirements connected to safety and comfort of the occupants. Predicting the structural dynamic behavior is one of the most difficult tasks, especially when such big vehicles as urban buses are considered. Due to their size and complexity, experimental derivation of comprehensive dynamic performances can be very difficult and economically unjustified. However, as vehicle dynamics is a very important performance, it cannot be neglected in vehicle analyses. Therefore, there is an opportunity to take advantage of numerical modeling and simulations, to investigate structural performances under different operational conditions. However, despite that fact, there is a lack of engineering literature describing urban bus virtual prototyping projects. Such a literature would have been of help in understanding how to create models that allow accurately predicting the dynamic behavior of the bus. Standard vehicle design process provides short concept design phase and very long prototype testing stage. However, following van der Auweraer and Leuridan $[1,2]$, modern product development is often based on virtual prototyping according to the rule "Design Right First Time" [3]. It is caused by the need for decreasing production costs and time to market of new vehicle and improving the product quality at the same time.

The main objective for the engineering design of passenger vehicles is to develop models that perfectly reflect the behavior of a real construction. Typically, to obtain trustworthy structural dynamic response, modal analysis of a real structure has to be performed $[4,5]$. Modal analysis is carried out to extract structural resonance frequencies and spatial description of vibration patterns, called normal modes frequencies and shapes, respectively. Classical modal analysis is based on a controlled excitation of a system vibration and measurement of a structure response. This can be easily simulated with a standard finite element approach. However, due to the necessity of input forces measurements, classical formulation has a limited use for a lot of large structures like an urban bus, and it is more convenient to use a method based on operational excitation, called operational modal analysis [6]. Therefore, during the simulations, engineers try 
to recreate vehicle operating conditions to obtain the most accurate results.

Virtual test rides can be modeled with multibody techniques. Multibody models are used to model the dynamic behavior of a vehicle, which may undergo large translational and rotational displacements. For this reason, those models can be used to simulate vehicles rides. Unfortunately, many problems connected to dynamics cannot be solved by using rigid body approximation of the structure. The necessity for more reliable models describing the complex behavior of mechanical systems undergoing large motions with small elastic deformations forced the development of many powerful analysis techniques. One of the most popular is coupling of rigid multibody systems and flexible components (mostly finite element models), supplemented by auxiliary 1D systems [7].

For this reason, the authors decided to build a hybrid urban bus model that was composed of a rigid-flexible structural multibody model, equipped with on-board intelligent systems that have an influence on riding conditions and, consequently, on the results of dynamic analyses.

The developed model can be used to evaluate a structure's behavior during virtual test rides, which can be carried out on test lanes providing desired dynamic excitations and forcing different maneuvers.

In this paper, an introduction to rigid-flexible body coupling is provided in the beginning. The subsequent parts describe the link between 3D spatial model and block 1D modeling of the on-board systems and the model itself. Such a link is called cosimulation and is an innovative technique that allows performing simulations from different engineering domains (from vehicle dynamics to NVH analysis) in shorter time and in automatic way. The paper describes hybrid urban bus modeling process with underlined base model parts like suspension, structure, and on-board systems. Simulation results are then correlated with experimental data showing correctness of the technique. Those chapters are then followed by the discussion and conclusions.

\section{Coupled Rigid-Flexible Multibody Model}

Due to the fact that the full model has been developed with LMS Virtual.Lab software, rigid and flexible equations of motion are provided based on [8].

Multibody modeling technique has been developed to simulate industrial and technological applications that are made of interconnected components which exhibit relative movement. These components are represented by sets of bodies linked together by joints and coupling elements like springs, dampers conjugated with constraints, and external force components. The system has to be prepared in order to allow motion in the desired direction to ensure required operating conditions (Figure 1).

Rigid multibody systems are considered to have a fixed frame of reference in which motion of the chosen points is observed.

To describe rigid multibody motion as a function of time, generation of equations of motion is needed. However, for

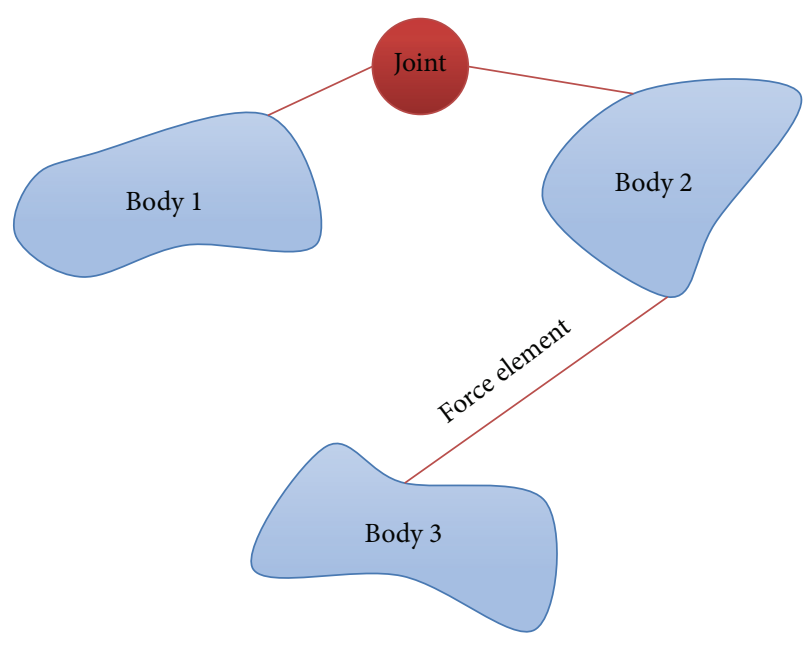

FIGURE 1: Multibody system.

large systems, it is a nontrivial task. For this reason, a lot of formalisms have been developed. Numerical formalisms especially play a significant role as they are used in computer multibody codes which becomes more and more popular and sophisticated [9].

The most popular formalism is one based on NewtonEuler equations rewritten in the matrix form and used in LMS Virtual.Lab [8]:

$$
\left[\begin{array}{cc}
M & \Phi_{q}^{T} \\
\Phi_{q} & 0
\end{array}\right] \cdot\left[\begin{array}{c}
\ddot{q} \\
1
\end{array}\right]=\left[\begin{array}{l}
Q \\
g
\end{array}\right],
$$

where $M$ represents a multibody mass matrix, $\Phi_{q}$ is a Jacobian matrix of the vector of constrains relative to the generalized coordinates $q$, and $Q$ is a vector of applied forces while $g$ is the constraint acceleration gamma term and $\lambda$ is the vector of Lagrange multipliers of the constraints. Equation (1) is then solved inside Virtual.Lab with special integration codes.

Unfortunately, many dynamic problems cannot be solved using only rigid body formulations. In case of small deformations, especially, to calculate structural dynamics, the linear finite element modeling (FEM) method is employed. For this reason coupling between multibody systems and finite element models has become an issue. Finite element models represent linear elastic deformation and can be integrated with nonlinear, large rotations and translations (multibody models). Different solutions for flexible multibody systems can be found in [10].

In Virtual.Lab formulation, the coupled flexible-rigid multibody models require a finite element analysis. The flexibility is represented with a set of flexible body modes which is a combination of normal modes (to represent natural vibration) and static ones (to represent loading and coupling between bodies). 


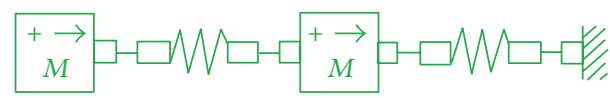

FIgURE 2: Example of 1D model (block-diagram).

Equation of motion with applied flexibility leads to

$$
\left[\begin{array}{ccc}
M_{r} & M_{r f} & \Phi_{q r}^{T} \\
M_{f r} & M_{f} & \Phi_{q f}^{T} \\
\Phi_{q r} & \Phi_{q f} & 0
\end{array}\right] \cdot\left[\begin{array}{c}
\ddot{q}_{r} \\
\ddot{u} \\
\lambda
\end{array}\right]=\left[\begin{array}{c}
Q_{r} \\
Q_{f} \\
g
\end{array}\right] .
$$

Mass matrix $M$ is divided into $r$-rigid part and $f$-flexible part; the same applies for Jacobian matrix $\Phi$ of the constraint equations. Apart from rigid generalized coordinates $q$, a vector of modal coordinates $u$ appeared; $\lambda$ represents Lagrange multiplier. On the right-hand side, vector of applied forces $Q$ divided into $Q_{r}$ forces applied on a rigid body and $Q_{f}$ forces applied on a flexible body (including body stiffness matrix) and general constrain force $g$ are shown.

The mentioned set of flexible body modes can be obtained in a large number of methods [8]. One of them is the CraigBampton dynamic reduction [11]. This method combines normal modes with static-constraint modes $\left(q, u_{b}\right)$ by CraigBampton transformation matrix $\Phi_{\mathrm{CB}}$ which contains a fixed base modeshape $\Phi_{L}$ and a rigid body vector $\Phi_{R}$ :

$$
u=\Phi_{\mathrm{CB}} \cdot\left\{\begin{array}{c}
u_{b} \\
q
\end{array}\right\}=\left[\begin{array}{cc}
I & 0 \\
\Phi_{R} & \Phi_{L}
\end{array}\right] \cdot\left\{\begin{array}{c}
u_{b} \\
q
\end{array}\right\}
$$

Modern coupling techniques are still under investigation.

\section{1D System Simulation}

The one-dimensional modeling is a mathematical approach in representation of components. In contrast to $3 \mathrm{D}$ CAE modeling, this approach gives the capability to simulate the behavior of systems before detailed CAD geometry is available. Model components are described using validated analytical models that represent the systems behavior. This technique of modeling is also referred to as a block modeling or a multiport modeling technique [12]. 1D models are physical models of real systems that are described with mathematical models (systems equation of motion).

One-dimensional models are created by linking individual physical elements together, creating a block diagram of full system (Figure 2). In such a system, the signal flows between individual components. Due to different mathematical representations of individual elements, the input signal is transformed in a predefined manner, to form an output. Those $1 \mathrm{D}$ models can be treated separately (solving their equations of motion) or can be linked with 3D models by cosimulation method linking the two solvers (1D and 3D) that can run separately by embedding one set of equations into the other, using only one single solver.

This solution is widely used among automotive engineers especially to simulate electronic systems inside a vehicle [13].

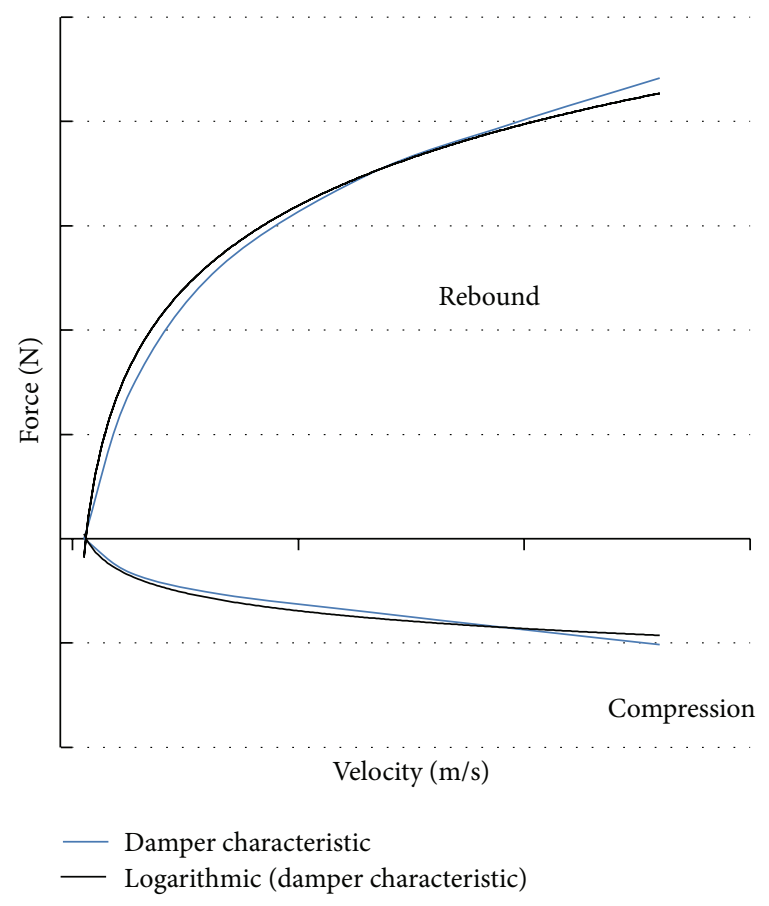

FIgUre 3: Typical damper characteristic and its logarithmic approximation.

One platform that provides a possibility to build $1 \mathrm{D}$ models is LMS Imagine.Lab AMESim that is used in this work.

\section{Hybrid Urban Bus Model}

4.1. Coupled Bus Multibody Model. The urban bus model developed at AGH UST has been based on a real city bus construction. Individual components have been modeled using CAD geometry and characteristics and additional information provided by the manufacturer. The model contains about 70 individual parts and about 90 kinematic joins. The number of general coordinates (including modal coordinates) is less than 500 .

In case of structural dynamics behavior, the suspension is used only as a filter between the track and the bus superstructure. Because of a high rigidity of the suspension structural elements and the frequency band of interest, their deformations were neglected. For this reason, all suspension elements, excluding tires, have been prepared as rigid bodies. Only coupling elements (dampers and bushings) have been described with original characteristics to incorporate proper stiffness and damping characteristics to the model. To obtain accurate damping elements characteristics for the front and the rear suspension, a logarithmic approximation has been performed according to the following (Figure 3 ):

$$
F=c \cdot \ln (v) .
$$

$F$ represents a rebound/compression damper force, $c$ is a constant (depends on damper type), and $v$ is a car body vibration velocity relative to the wheels. 
To connect bus suspension elements, based on supplier technical data, bushing elements are used. Those elements provide an interface between two parts, damping the energy transmitted through the bushing. Bus suspension bushings are made of rubber separating the faces of two metal objects while allowing a certain amount of movement.

To provide good simulation results, bus suspension bushings have been modelled inside Virtual.Lab Motion software as special connector element called bushing. This element allows providing real bushing characteristics to the virtual model as an excel file.

Such connectors minimize vibrations through the chassis of the vehicle in virtual simulations like in the real vehicle.

Another important element that provides traction between the vehicle and the road and absorbs shock is the tire. Based on available information about tire characteristics from the bus manufacturer, those components have been modeled using a Virtual.Lab special tire module called Complex Tire, which permits inputting tire parameters like diameter, stiffness, damping, and so forth, in terms of providing real ride conditions. Parameters values have been taken from supplier data and adjusted to the values that provide good correlation between real experiment and simulation.

Unfortunately, because of missing measurement of real bushing and tires, no correlation directly for those elements has been done.

Urban buses are often equipped with a pneumatic suspension where standard steal springs are replaced with air cushions, filled with pressurized air. The main advantages of using air springs over the standard steel-spring suspensions in an urban bus are

(i) high comfort expressed by small deflections and lower natural frequencies,

(ii) possibility of controlling the operation conditions, by modifying the internal pressure value,

(iii) kneeling function-in case of buses this function eases getting on and off the vehicle on stops [14].

Air springs have been modeled as combined damping and stiffness forces elements controlled by ECAS (electronically controlled air suspension) system. Such a configuration provided a possibility of applying real air spring characteristics to the model.

Due to the fact that the scope of the investigations was to measure bus driver's comfort by means of structure vibration level, the bus superstructure has been modeled as a deformable element. Nastran finite element coding was used to create a FE model based on CAD geometry. To simplify the model, some of the elements like engine or gear have been substituted with concentrated mass elements. Figure 4(a) presents an urban bus suspension multibody model and Figure 4(b) presents a coupled flexible-rigid urban bus model.

4.2. Bus On-Board Systems. To ensure the most realistic simulation conditions and obtain the most accurate response of the analyzed urban bus, electronic auxiliary systems have also been taken into consideration. Nowadays, vehicles are equipped with electronic devices which improve ride quality and safety. For a regular urban bus, the most important are ABS/ASR and EBD (i.e., anti-lock braking system/antislip regulation and electronic breaking force distribution) to maintain safety during breaking maneuvers and ECAS (i.e., electronically controlled air suspension) system which controls the work of a pneumatic suspension [15]. To assure a realistic prediction of the behavior of the bus in operating conditions, the abovementioned systems have been applied to the coupled multibody model.

As it was mentioned before, bus structure and suspension have been modeled with 3D models (multibody and FE models), which is necessary to simulate its dynamic behavior. In case of on-board systems, it is not necessary to create 3D models of electronic devices as the most important thing is signal flow from the bus to the electronic units and vice versa. For this reason, on-board systems have been modeled with $1 \mathrm{D}$ block diagram method in Imagine.Lab AMESim software. To integrate 3D bus model and $1 \mathrm{D}$ electronic devices, a cosimulation between Virtual.Lab and AMESim has been used to exchange information between 3D and 1D models.

Such a cosimulation technique allows reducing simulation time, increasing the realism of the virtual bus.

A brake assist (ABS/ASR and EBD) block diagram, coupled with other parts of the model, is shown in Figure 5. In this circuit, predefined ABS/ASR and EBD elements have been used. The characteristic parameters have been elaborated according to the data provided by the systems manufacturers. The principles of the breaking maneuver, used for the simulation of the model equipped with electronic elements, are similar to realistic driver behavior. Multibody solver sends a signal to AMESim, which contains information about bus actual travelling velocity and angular velocities of each wheel. Based on those, breaking torque for each wheel is evaluated and resent to the leading model. Figure 6 shows that, with break assistant system, the angular velocity of the wheels is a nonzero value, while, without it, axes are blocked in slippage. This underlines the importance of this system for the passenger's safety.

The second system applied to a coupled multibody model was ECAS. Figure 7 presents the ECAS numerical model. This circuit has been fully developed at AGH UST. The investigated system has been equipped with 6 air cushions, two connected to the front axle and four supporting the rear one. This asymmetry is due to the unequal mass distribution - that is, engine, gearbox, and auxiliary systems are located at the back of the vehicle.

The ECAS system has been modeled using the inputs provided by the bus manufacturer. By virtue of submitted data, there was a need for approximation of the received numbers. The derivation of the mathematical models describing the dynamical behavior of the air spring can be found in [1620]. In general, if isothermal process is assumed inside the air spring, its static stiffness can be described by the following [18]:

$$
k_{s}=\frac{d F}{d x}=P \frac{A_{e}^{2}}{V}+\left(P-P_{a}\right) \frac{d A_{e}}{d x},
$$




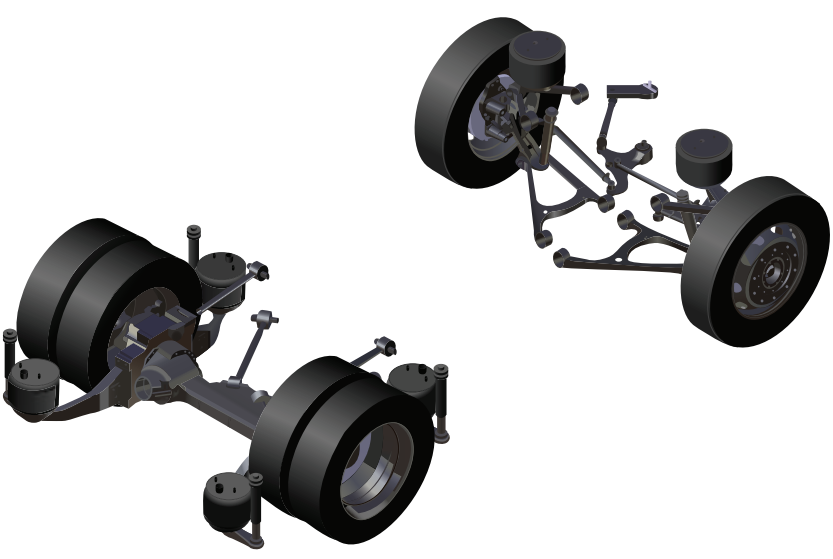

(a)

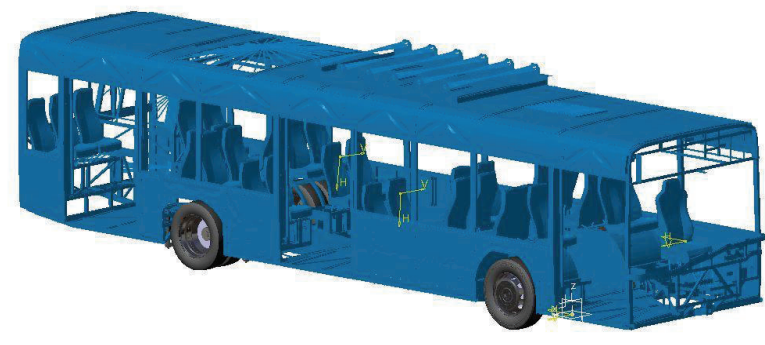

(b)

FIGURE 4: (a) Multibody bus suspension system; (b) rigid-flexible multibody bus model.

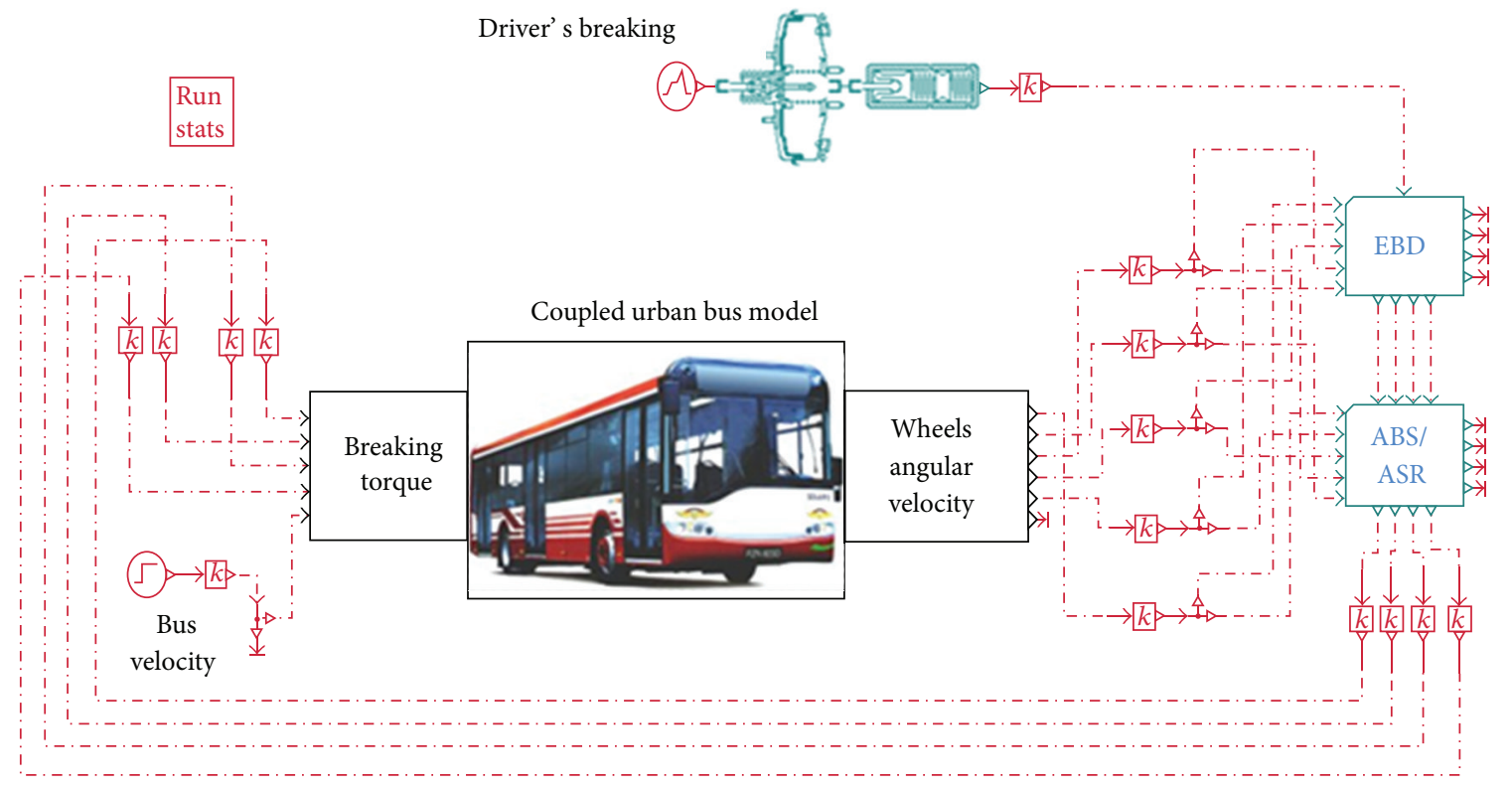

FIGURE 5: ABS/ASR/EBD signal circuit.

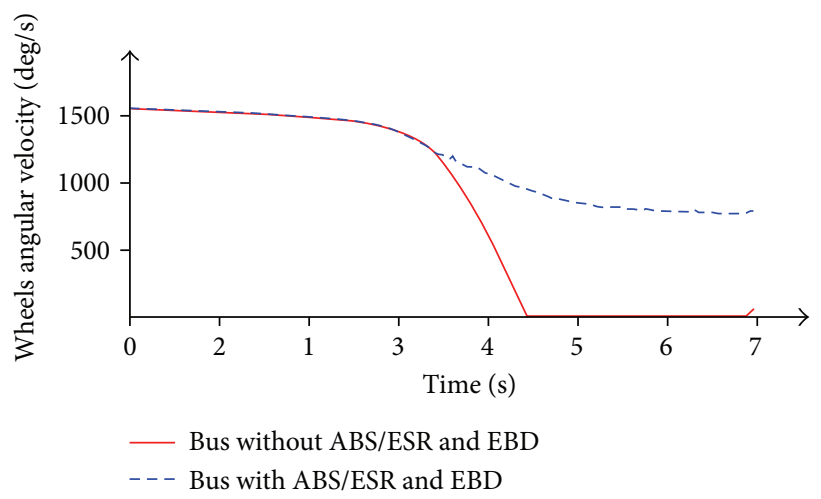

FIGURE 6: Virtual breaking performance on a slippery road. Comparison of angular velocity of the left wheel: with and without break assist. where $F$ is an applied force, $x$ is an air sprig deflection (difference between nominal and compressed air spring height), $P$ is the absolute pressure inside the air spring, $P_{a}$ is the atmospheric pressure, $V$ denotes internal bellows volume, and $A_{e}$ is an effective area of an air spring (i.e., supported load value divided by $P$ ).

Based on a manufacturer's technical data, a regression model approximating the static response of each air spring was developed. To fit a curve on the obtained measurement points, linear robust least squares method has been employed. Standard least squares method tends to minimize the squared distance between the regression curve and the provided data points; thus, it is sensitive to outliers. To omit that problem, algorithm described by (6) [17] was implemented which assigns a weighting factor to every measurement point. The value is inversely proportional to the distance of the measured 


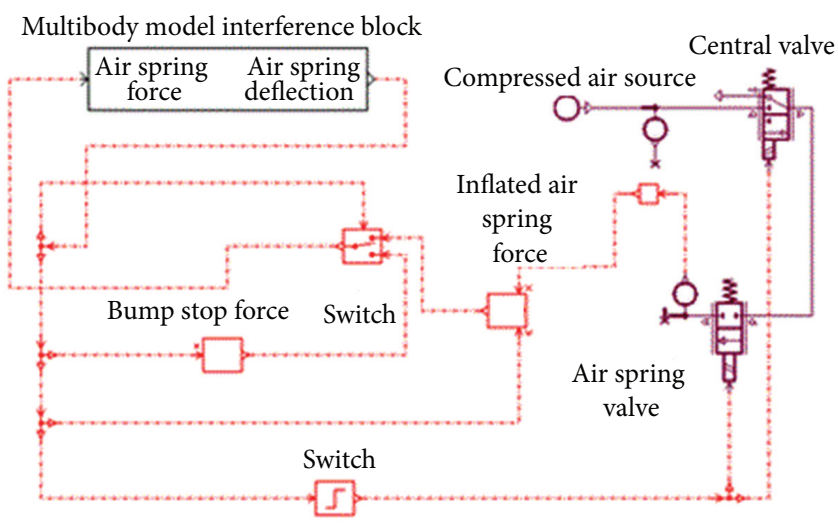

FIGURE 7: One-quarter of an ECAS model.

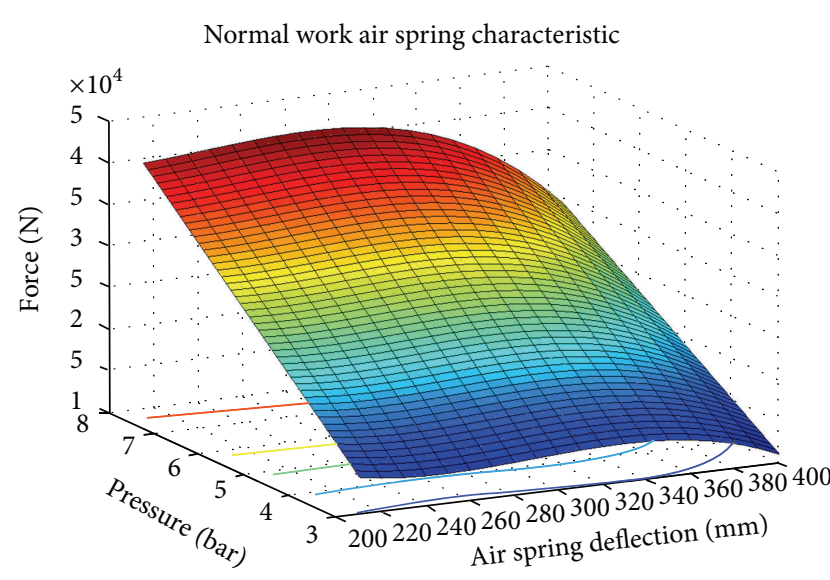

(a)

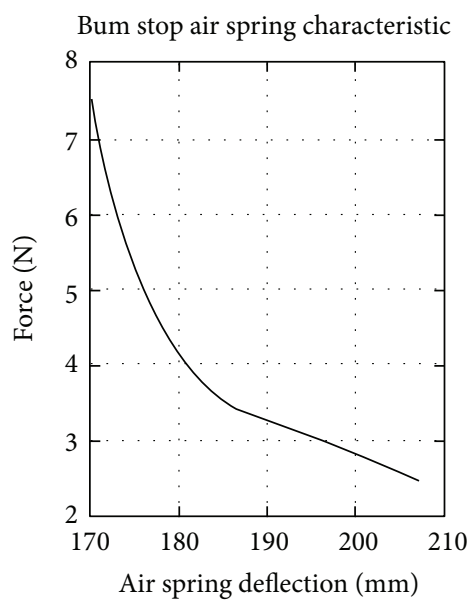

(b)

FIGURE 8: Measurement data approximation: the inflated air spring (a) and the bump stop (b) characteristics.

points from the fitted polynomial curve; thus, the influence of the outliers can be minimized. Furthermore, points which are further than would be expected are ignored during the regression process. Consider

$$
S=\sum_{i=1}^{n} w_{i}\left(y_{i}-\widehat{y}_{i}\right)^{2}
$$

where $w_{i}$ is the weighting factor for the squared distance between measured $y_{i}$ and fitted $\widehat{y}_{i}$ values. Measuring equipment inaccuracies are supposed to be invariant in time; hence, the variance of the acquired data should be constant. If this assumption is violated, it is highly probable that input data set contains some elements of poor quality. The weights $w_{i}$ are then applied to transform the variances to a constant value. Results of approximation are visible in Figure 8. Because of a strong nonlinearity of the abovementioned characteristic and for simplification, the characteristic was split into two separate regions: force generated by an inflated air spring and force generated by a bump stop, after reaching a critical deflection. The latter is expressed only as a function of bump stop material stiffness.
Consistent with a measured air spring deflection (received from a multibody model) and desired pressure, corresponding force value is sent back to the base structure. If the deflection falls below the specified value, the signal is switched to the bump stop characteristics. If, on the other hand, operational height is within the allowable tolerances, compressed air flow is cut off and the air spring is working as an inflated cushion.

Based on [18], the authors have introduced also an appropriate damping coefficient to the analyzed bus model to simulate not only air spring stiffness but also its damping behavior.

Coupling between flexible and rigid bodies and link with on-board systems block diagrams ensures real ride conditions. Because of a juncture between different modeling techniques, such a structure is called a hybrid model.

\section{Simulations}

The hybrid model described in a previous chapter has been developed to perform structural dynamics analyses under operational conditions. 


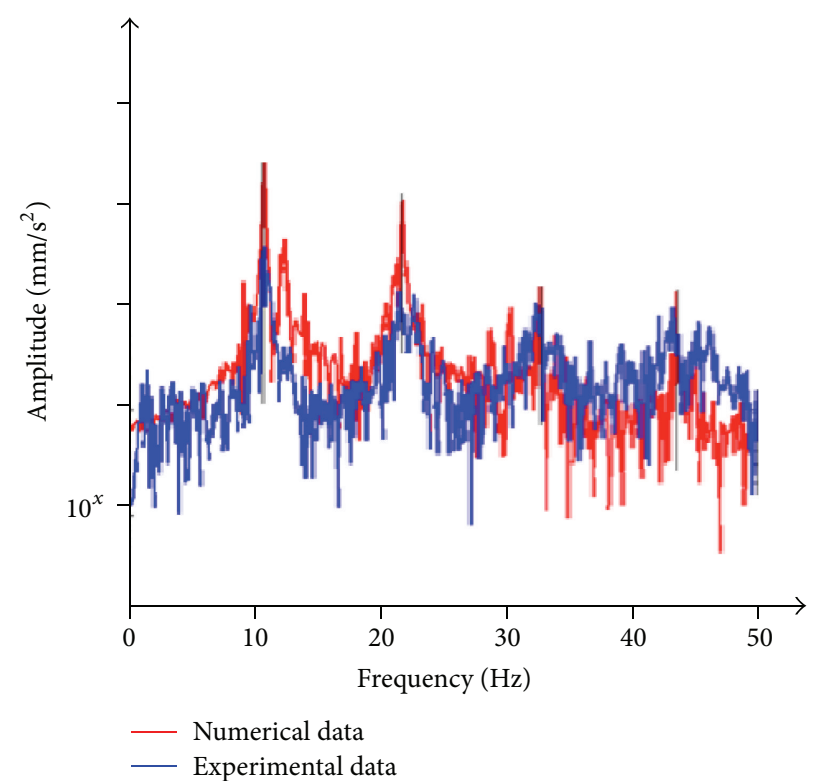

(a) Frequency response-point $\mathrm{A}$

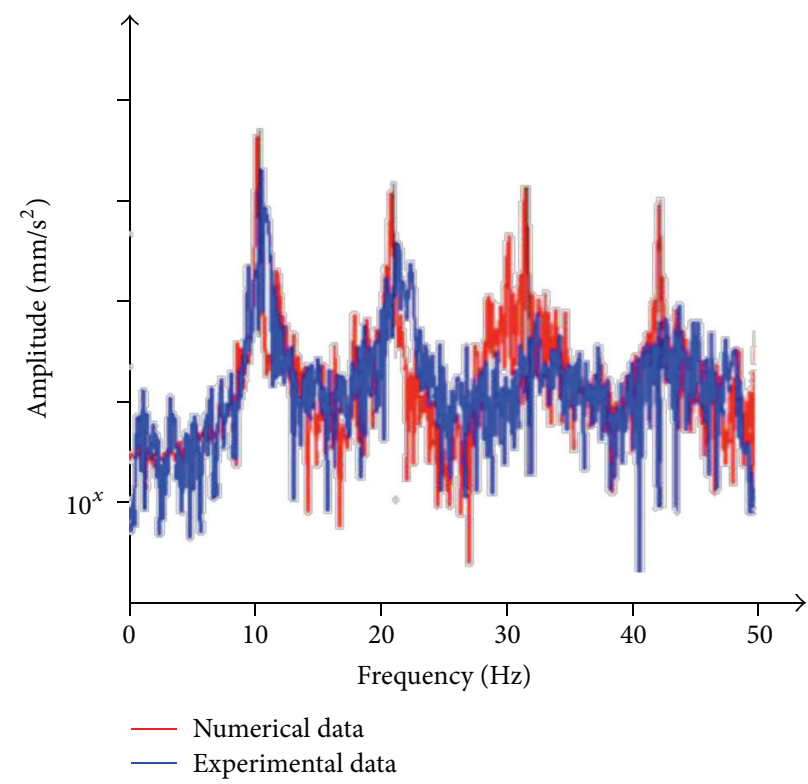

(c) Frequency response-point C

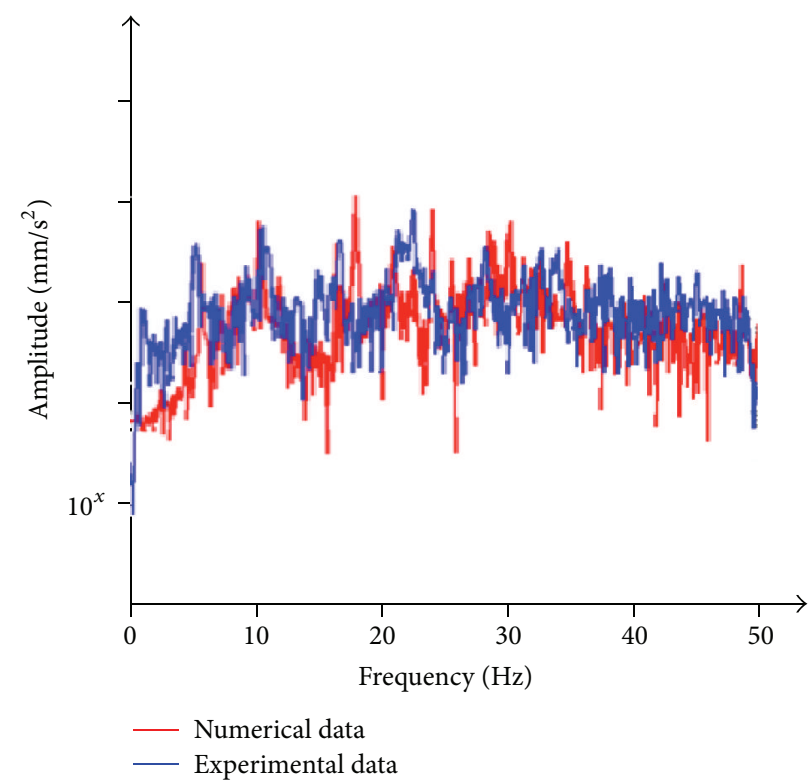

(b) Frequency response-point B

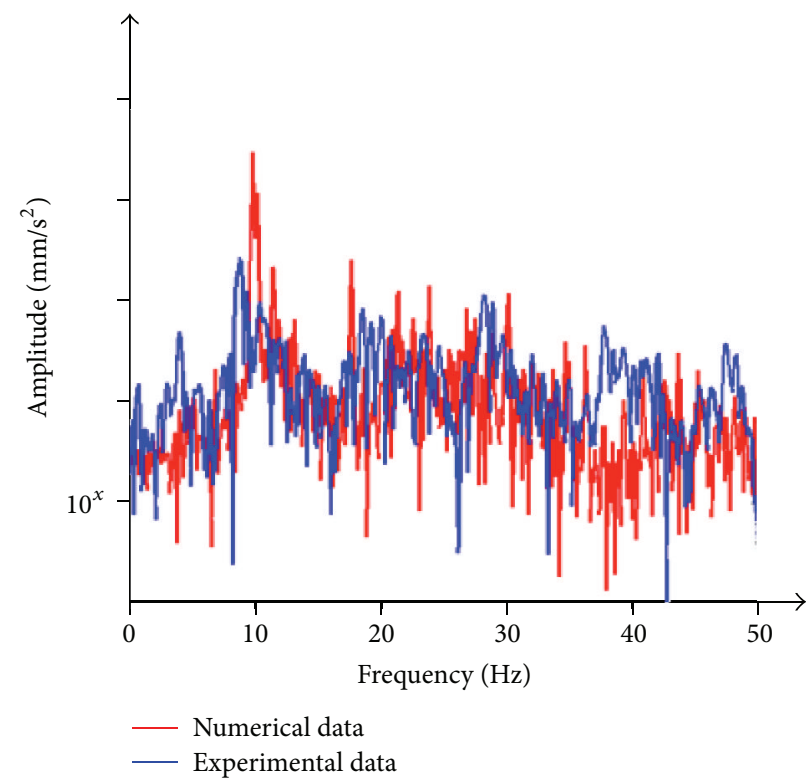

(d) Frequency response-point D

FIGURE 9: Comparison between numerical and experimental vibration acceleration as a function of frequency: (a) point A; (b) point B; (c) point $C$; (d) point $D$.

In order to examine a usefulness of the developed model for the structural measurements, virtual test has been performed and compared with the real test ride on a test track, which was combined from two different surfaces on its left and right side. To obtain the needed data, the conditions of the analysis were set the same as in the case of experiment; that is, the traveling speed was set to $30 \mathrm{~km} / \mathrm{h}$. During the experiment, the driver was obliged to keep the velocity constant, but, because of many factors (e.g., speedometer inaccuracy, irregular pavement conditions, etc.), this was only a rough value. Nevertheless, as can be seen in Figure 9, the results of numerical computations are in great correlation with the data obtained experimentally. The peaks at $11 \mathrm{~Hz}$ and their harmonics are the consequence of the form of the test track surface, which was covered by regularly distributed curbstones, and the vehicle's speed. Other peaks are caused by structural dynamic response.

Several measurement points located near the suspension mountings (points A (Figure 9(a)) and B (Figure 9(b))) and near the driver seat (points $\mathrm{C}$ (Figure 9(c)) and D (Figure 9(d))) were chosen to collect the experimental data during the tests. The obtained results were compared with the output from the numerical computations, which is shown in the examples in Figure 9. 


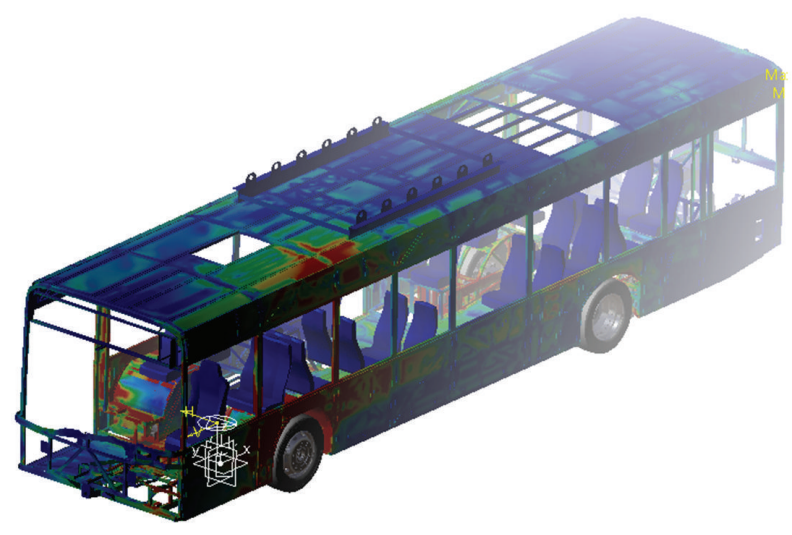

FIgURE 10: Hybrid urban bus model-stress Von Mises's measurement.

The differences are results of imprecise mapping of the road due to its complicated shape. Inaccuracies can also result from differences in placing the accelerometers on real and virtual structures, which affects the output data strongly. Another reason of the numerical and experimental data variations can arise from the tire modelling technique. As mentioned before, the tires have been modelled with the specialized Virtual.Lab module, including proper features like stiffness and damping. However, due to the complexity of tire dynamic characteristics and the difficulties in modelling the contact between the track and the wheels, those important parameters can be only roughly approximated.

However, the developed model is precise enough to be used for different measurements. For instance, the described model can be used for stress and strain evaluation; hence, the weakest regions can be pointed out. Figure 10 shows an example of a stress distribution map. Such analyses are extremely useful because they can reveal structural defects in the very first phase and, hence, the problem can be solved before the first prototype is released.

\section{Conclusions}

Urban buses have not yet been investigated thoroughly which opens the way to many improvements that can be obtained cheaply, by means of numerical methods. The presented hybrid urban bus model is a link between different modeling techniques which takes advantage of them in order to solve complex structural dynamics problems. Utilization of coupled flexible-rigid multibody model instead of dynamic loads on finite element model only gives more reliable representation of reality. Buses work on the streets and theirs structures are extorted with road irregularities. Applying such load conditions to a pure FE model is not a trivial task. However, with the proposed model, it can be achieved using an arbitrary track model and a contact connection with tires. Hybrid urban bus model has been developed, linking FE structure model (to make stress calculation on it possible), rigid suspension multibody model (for vehicle dynamics simulations with large translations and rotations), and 1D modeling techniques of on-board electronic devices (where time-consuming 3D modeling is not necessary). Such a model is useful for analyses of structure behavior under real, operational loads and optimizing in terms of vehicle parameters.

Such a model has been simulated in terms of operational conditions from real bus test on test truck. Results of simulation and real experiments have been compared. The comparison presented good correlation between data, which means that model can be used for virtual prototyping of urban buses.

The proposed multibody hybrid model can be used instead of FE representation, ensuring more precise results; due to application of realistic operating conditions, the usage of commercial software ensures the availability of the method.

The elaborated modeling technique can be also used in the optimization problems, combining both structural and vehicle dynamics, which is planned for the future.

\section{Conflict of Interests}

The authors declare that there is no conflict of interests regarding the publication of this paper.

\section{Acknowledgments}

This work is a part of international project EUREKA E! 4907 done at AGH University of Science and Technology from Poland with consortium from Belgium LMS International and Poland including EC Engineering and Solaris Bus \& Coach. The authors would like to gratefully acknowledge the support from the Polish National Centre of Research and Development. The authors kindly acknowledge also the other researchers at LMS International, EC Engineering, and SOLARIS Bus \& Coach, who have contributed to the CHASING R\&D activities and results reported in this paper.

\section{References}

[1] H. van der Auweraer and J. Leuridan, "A new testing paradigm for today's product development process-part 1," Sound and Vibration, vol. 39, no. 9, pp. 14-18, 2005.

[2] H. van der Auweraer and J. Leuridan, "A new testing paradigm for today's product development process-part 2," Sound and Vibration, vol. 39, no. 11, pp. 18-22, 2005.

[3] S. Donders, Computer-aided engineering methodologies for robust automotive NVH design [Ph.D. dissertation], KU Leuven, 2008.

[4] T. Uhl, "Computer aided model identification of mechanical systems," WNT, 1997.

[5] R. Craig, Structural Dynamic, John Wiley \& Sons, 1981.

[6] T. Uhl and W. Lisowski, "Operational modal analysis," Wydawnictwo Katedry Dynamiki Maszyn i Robotyki AGH w Krakowie, 1999 (Polish).

[7] A. Shabana, Dynamic Analysis of Large-Scale Inertia Variant Flexible Systems [Ph.D. dissertation], University of Iowa, 1982.

[8] LMS, Virtual.Lab online help, 2012.

[9] W. Schiehlen, Multibody Systems Handbook, Springer, 1990.

[10] A. Shabana, Dynamics of Multibody Systems, Cambridge University Press, 2005. 
[11] MSC.Software, MSC.Nastran 2001: Superelement User's Guide, 2001.

[12] LMS, "AMESim platform," 2012, http://www.lmsintl.com.

[13] A. Sarniotti and G. Repici, "A simulation based approach to failsafe systems in automotive design," in WSEAS International Conferences, Miami, Fla, USA, January 2004.

[14] WABCO, Electronic level control (ECAS) for buses with pneumatic suspension system, 1997, (Polish), http://inform.wabcoauto.com.

[15] L. Prochowski and A. Żuchowski, Trucks and Buses, Wydawnictwo Komunikacji i Lacznosci, 2004.

[16] D. Ha, H. Kim, and H. Lee, "Height sensor fault diagnosis for electronic air suspension (EAS) system," in Proceedings of the IEEE International Symposium on Industrial Electronics (ISIE '09), pp. 211-216, Seoul, Republic of Korea, July 2009.

[17] Engineering Statistic Handbook, http://www.itl.nist.gov/div898/ handbook/pmd/sectionl/pmd143.htm.

[18] H. Liu and J. Lee, "Model development of automotive air spring based on experimental research," in Proceedings of the 3rd International Conference on Measuring Technology and Mechatronics Automation (ICMTMA '11), vol. 2, pp. 585-590, Shanghai, China, January 2011.

[19] H. Kim and H. Lee, "Height and leveling control of automotive air suspension system using sliding mode approach," IEEE Transactions on Vehicular Technology, vol. 60, no. 5, pp. 20272041, 2011.

[20] I. Jang, H. Kim, H. Lee, and S. Han, "Height control and failsafe algorithm for closed loop air suspension control system," in Proceedings of the International Conference on Control, Automation and Systems (ICCAS '07), pp. 373-378, Seoul, Republic of Korea, October 2007. 

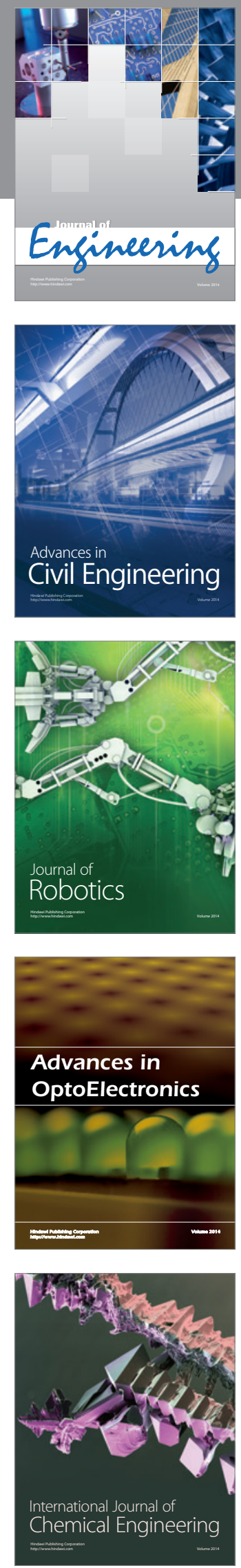

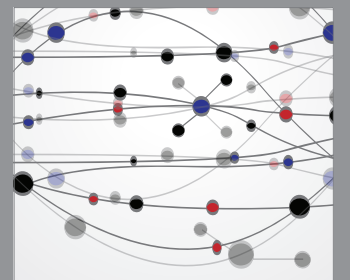

The Scientific World Journal
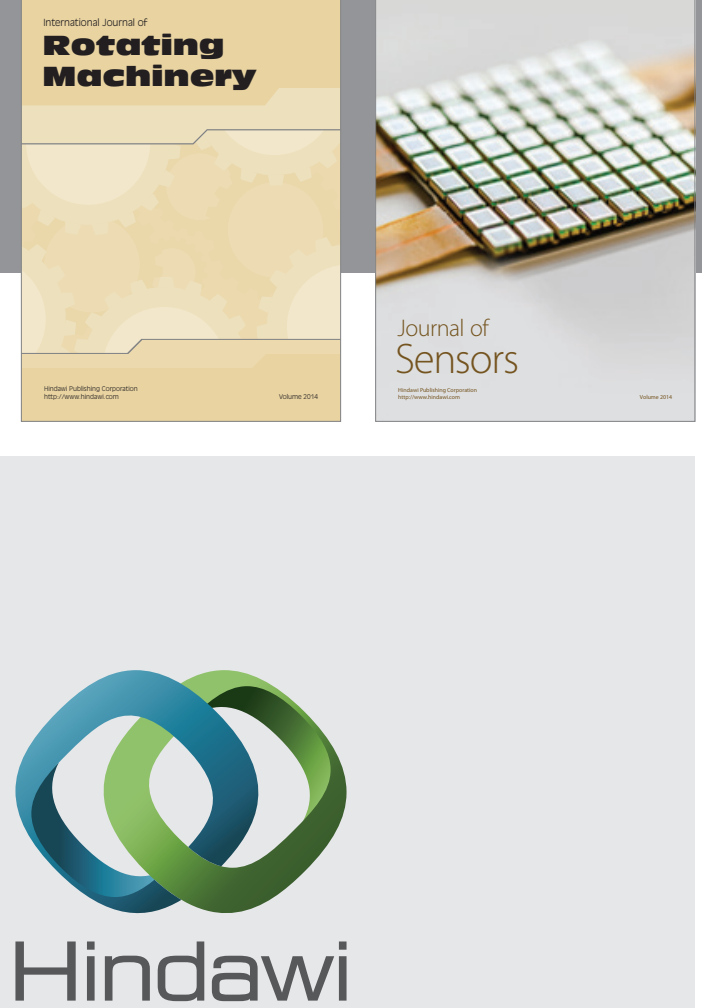

Submit your manuscripts at http://www.hindawi.com
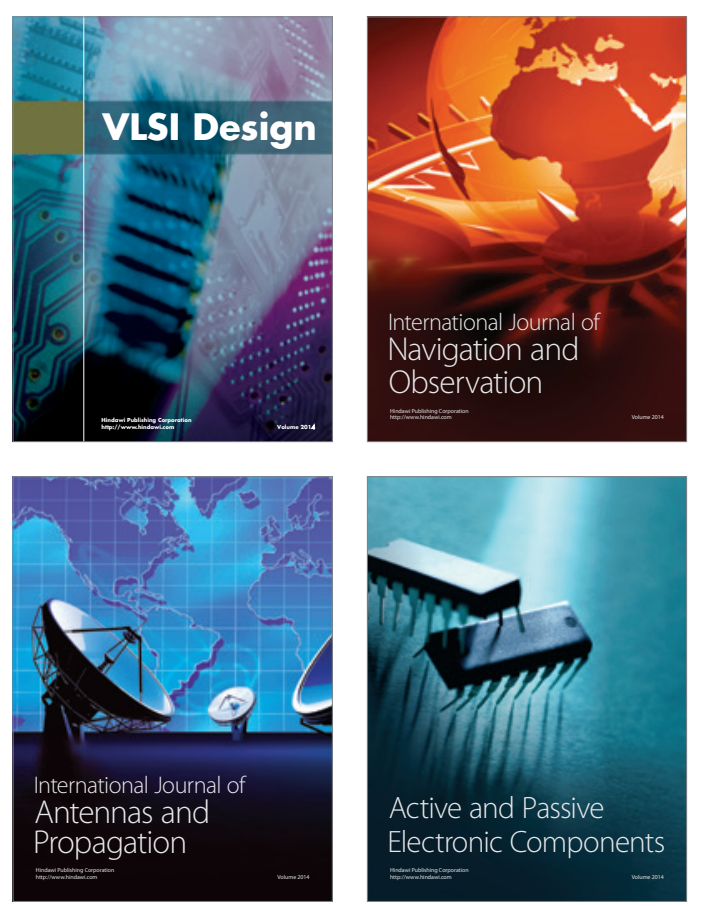
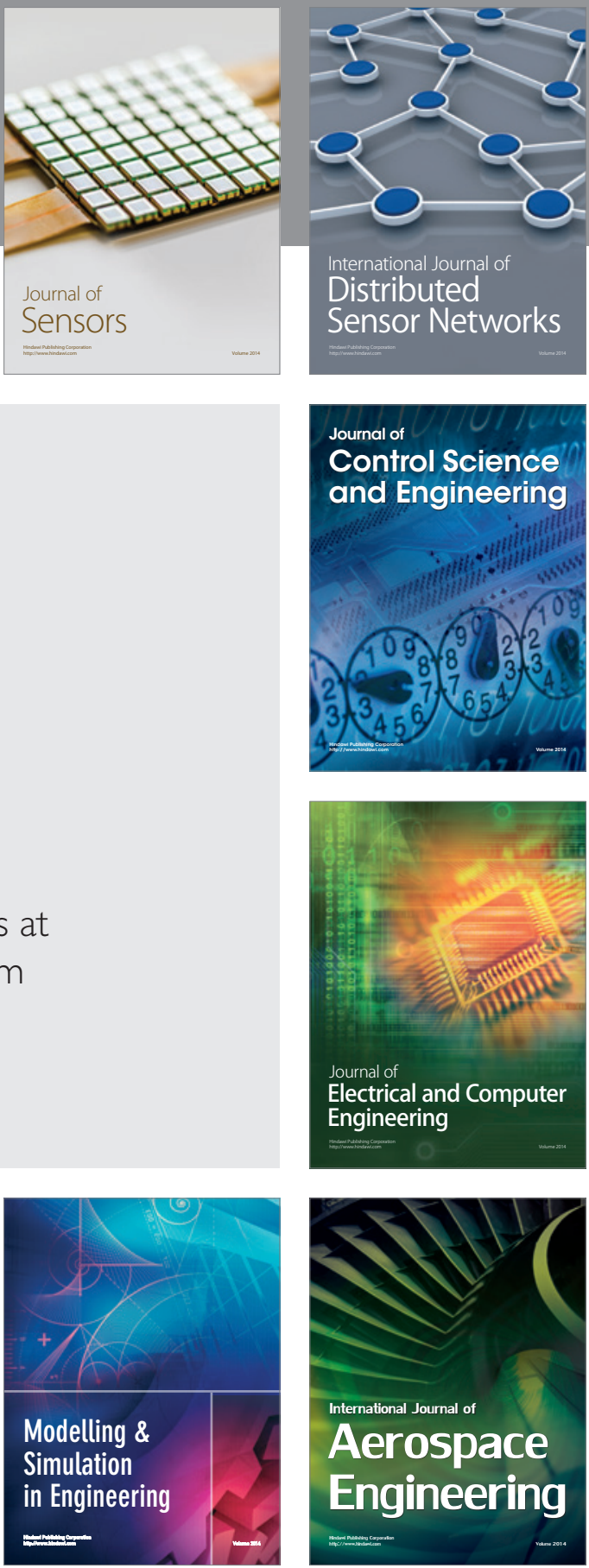

Journal of

Control Science

and Engineering
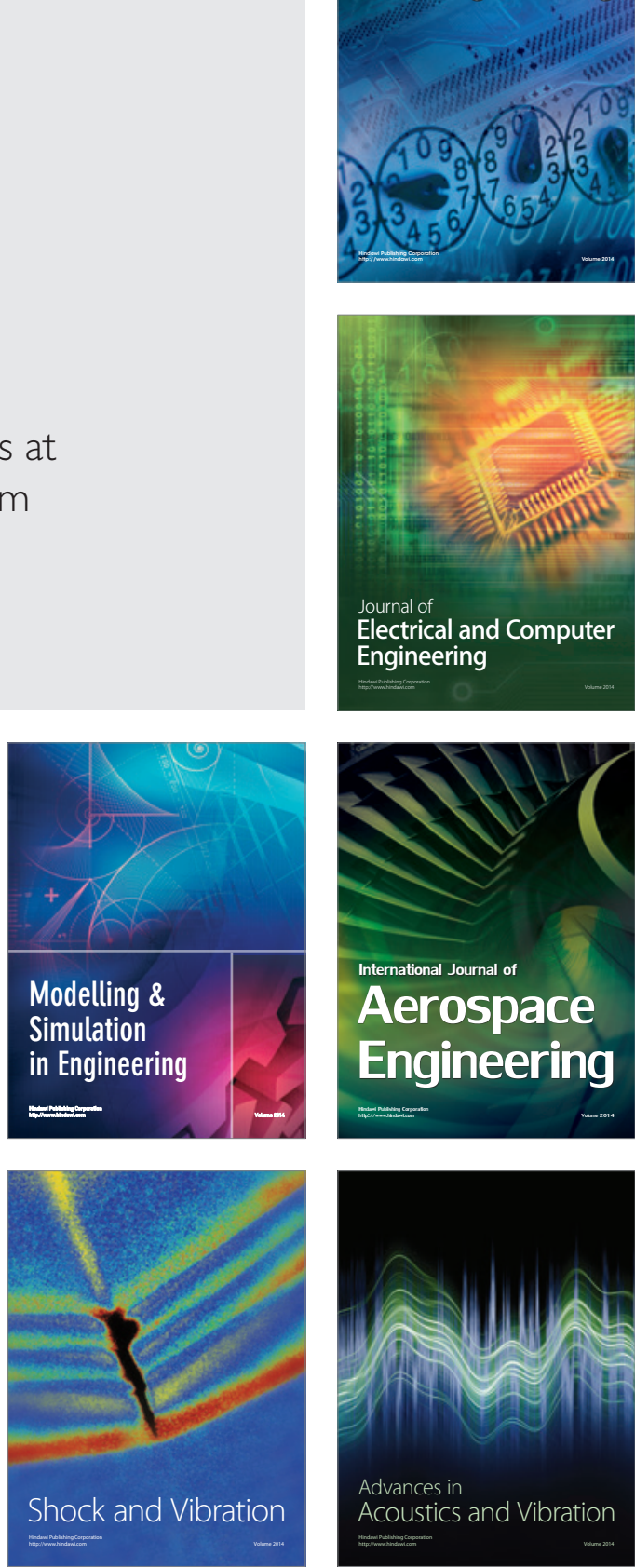